\title{
Higher-Order Modulation Instability in Nonlinear Fiber Optics
}

\author{
Miro Erkintalo, ${ }_{1}$ Kamal Hammani, ${ }^{2}$ Bertrand Kibler, ${ }^{2}$ Christophe Finot, ${ }^{2}$ Nail Akhmediev, ${ }^{3}$ \\ John M. Dudley, ${ }^{4}$ and Goëry Genty ${ }^{1}$ \\ ${ }^{1}$ Tampere University of Technology, Optics Laboratory, FI-33101 Tampere, Finland \\ ${ }^{2}$ Laboratoire Interdisciplinaire Carnot de Bourgogne, UMR 5209 CNRS/Université de Bourgogne, 21078 Dijon, France \\ ${ }^{3}$ Research School of Physics and Engineering, The Australian National University, Canberra ACT 0200, Australia \\ ${ }^{4}$ Institut FEMTO-ST UMR 6174 CNRS/Université de Franche-Comté, Besançon, France
}

(Received 29 July 2011; published 13 December 2011)

\begin{abstract}
We report theoretical, numerical, and experimental studies of higher-order modulation instability in the focusing nonlinear Schrödinger equation. This higher-order instability arises from the nonlinear superposition of elementary instabilities, associated with initial single breather evolution followed by a regime of complex, yet deterministic, pulse splitting. We analytically describe the process using the Darboux transformation and compare with experiments in optical fiber. We show how a suitably low frequency modulation on a continuous wave field induces higher-order modulation instability splitting with the pulse characteristics at different phases of evolution related by a simple scaling relationship. We anticipate that similar processes are likely to be observed in many other systems including plasmas, Bose-Einstein condensates, and deep water waves.
\end{abstract}

DOI: 10.1103/PhysRevLett.107.253901

PACS numbers: 42.65.Tg, 42.65.Re, 42.65.Sf, 42.81.Dp

The Benjamin-Feir or modulation instability (MI) is a central process of physical systems described by the focusing nonlinear Schrödinger equation (NLSE). MI dynamics are associated with energy exchange between a periodic perturbation and a continuous background, and have been extensively studied since the 1960s [1]. Despite this long history, MI is once again the subject of significant interest as a mechanism to describe the emergence of strongly localized "rogue wave" structures in hydrodynamics and optics [2-4]. Of particular importance has been the realization that many properties of MI previously described only approximately (via numerical or truncated mode approaches) can in fact be described almost exactly using known analytic NLSE Akhmediev-breather solutions [5]. This has led to successful studies of the spectral characteristics of MI excited from noise [6] and experiments exciting the previously unobserved Peregrine soliton limit of the NLSE [7-9].

For ideal initial conditions, NLSE breathers exhibit only one growth-return cycle. Such evolution, however, requires a precisely constructed initial field consisting of an infinite number of frequency modes with appropriate phases [10]. More realistic studies (both numerical and in physical systems such as optics) have shown that such ideal single growth-return evolution is not generally observed, but rather the initial phase of breather localization is followed by a regime of significantly more complex evolution [11]. Specifically, when the second harmonic of the initial modulation frequency falls under the elementary MI gain curve it has been shown both numerically [11,12] and experimentally [8] that the breather undergoes decomposition and splits into two subpulses. In this Letter, we show that these dynamics are in fact the manifestation of a particular regime of NLSE propagation associated with the excitation of higher-order modulation instability, and we present what is to our knowledge the first combined experimental, theoretical, and numerical study of this process in any NLSE-described system.

The defining physics of higher-order MI is the simultaneous excitation of multiple instability modes, each mode associated with a corresponding nonlinear breather. The rich dynamics that is observed then arises from the nonlinear superposition of the individual constituent breathers. Here, we demonstrate how the excitation of higher-order MI can be observed readily in experiments using only a single initial frequency modulation on a plane wave, provided that the modulation frequency is below a critical low frequency limit such that multiple instability harmonics fall under the elementary gain curve. We apply the Darboux transformation $[11,13]$ to analytically describe the complex splitting dynamics, and we show excellent agreement with numerical simulation and experiment. Another significant originality of our work is that it represents to our knowledge the first example in any NLSE system where the Darboux transformation is used for the analysis and interpretation of physical experiments, and thus our results validate this powerful analytical technique. In addition, insights from the Darboux transformation approach lead us to develop a simple scaling criterion relating pulse characteristics at various phases of the instability, making an important link with previous studies of self-similar dynamics in NLSE systems [14,15]. Finally, we note that because the Darboux transformation allows for an infinite number of new solutions to be constructed, its experimental validation opens up possibilities to observe a large class of novel highorder instabilities at very high nonlinearity, a regime that is 
generally intractable using numerical techniques. Our results could therefore represent a major step towards understanding extreme wave localization dynamics in nonlinear systems including plasmas, Bose-Einstein condensates, and deep water waves [11], as well as providing a theoretical framework for the interpretation of various regimes of nonlinear turbulence [16].

We first discuss the NLSE breather solutions and the Darboux transformation. The dimensionless focusing NLSE is

$$
i \frac{\partial \psi}{\partial \xi}+\frac{1}{2} \frac{\partial^{2} \psi}{\partial \tau^{2}}+|\psi|^{2} \psi=0,
$$

where $\xi$ and $\tau$ are normalized distance and time, respectively. The modulation instability dynamics of a harmonic perturbation on an initial plane wave are well described by the breather solution of Akhmediev [5]:

$$
\begin{aligned}
\psi(\xi, \tau)= & {\left[\frac{(1-4 a) \cosh \left[b\left(\xi-\xi_{0}\right)\right]+i b \sinh \left[b\left(\xi-\xi_{0}\right)\right]}{\cosh \left[b\left(\xi-\xi_{0}\right)\right]-\sqrt{2 a} \cos \left[\Omega\left(\tau-\tau_{0}\right)\right]}\right.} \\
& \left.+\frac{\sqrt{2 a} \cos \left[\Omega\left(\tau-\tau_{0}\right)\right]}{\cosh \left[b\left(\xi-\xi_{0}\right)\right]-\sqrt{2 a} \cos \left[\Omega\left(\tau-\tau_{0}\right)\right]}\right] e^{i \xi}
\end{aligned}
$$

Here $\Omega$ is a dimensionless frequency related to the parameter $a$ through $a=1 / 2\left(1-\Omega^{2} / 4\right)$, and the instability growth is $b=[8 a(1-2 a)]^{1 / 2}=\Omega\left(1-\Omega^{2} / 4\right)^{1 / 2}$. There is MI gain for $0<a<1 / 2$, corresponding to frequencies $\Omega_{\max }>\Omega>0$ with $\Omega_{\max }=2$. Real parameters $\xi_{0}$ and $\tau_{0}$ describe the spatial and temporal centers of the solution. Transformations to dimensional quantities in the context of fiber optics are given later when we describe our experiments.

The solution in Eq. (2) was originally obtained using a physically motivated ansatz [5], but similar solutions have been obtained using the inverse scattering technique $[17,18]$. Another powerful approach to NLSE analysis is the Darboux transformation, a linear algebraic dressing method allowing successive construction of higher-order solutions from a known solution [13]. The approach is based on representing the NLSE as the compatibility condition between the following linear set [11]:

$$
\begin{aligned}
& \mathbf{R}_{\tau}=-\mathbf{J R} \Lambda+\mathbf{U R}, \\
& \mathbf{R}_{\xi}=-\mathbf{J R} \Lambda^{2}+\mathbf{U R} \Lambda-\frac{1}{2}\left(\mathbf{J} \mathbf{U}^{2}-\mathbf{J} \mathbf{U}_{\tau}\right) \mathbf{R},
\end{aligned}
$$

where $\mathbf{R}, \mathbf{J}, \mathbf{U}$, and $\Lambda$ are $2 \times 2$ matrices defined by

$$
\begin{array}{ll}
\boldsymbol{\Lambda}=\left[\begin{array}{cc}
\lambda & 0 \\
0 & \lambda^{*}
\end{array}\right], \quad \mathbf{J}=\left[\begin{array}{cc}
i & 0 \\
0 & -i
\end{array}\right], \\
\mathbf{U}=\left[\begin{array}{cc}
0 & \psi \\
-\psi^{*} & 0
\end{array}\right], \quad \mathbf{R}(\sigma)=\left[\begin{array}{cc}
r(\sigma) & s^{*}(\sigma) \\
s(\sigma) & -r^{*}(\sigma)
\end{array}\right] .
\end{array}
$$

Here, the functions $r(\sigma)=r(\xi, \tau ; \sigma)$ and $s(\sigma)=s(\xi, \tau ; \sigma)$ depend on a set $\sigma$ of three arbitrary constants: the spectral parameter $\lambda$ and two integration constants. The compatibility condition $\mathbf{R}_{\tau \xi}=\mathbf{R}_{\xi \tau}$ is equivalent to Eq. (1), which means that the set of Eq. (3) is simultaneously satisfied for all $\Lambda$ if and only if $\psi(\xi, \tau)$ is a solution of the NLSE. The linear set (3) is invariant under the Darboux transformation:

$$
\begin{aligned}
\mathbf{R}(\sigma) & \rightarrow \mathbf{R}_{1}\left(\sigma_{1}, \sigma\right)=\mathbf{R}(\sigma) \Lambda-\kappa\left(\sigma_{1}\right) \mathbf{R}(\sigma), \\
\mathbf{U} & \rightarrow \mathbf{U}_{1}=\mathbf{U}-\mathbf{J} \kappa\left(\sigma_{1}\right)+\kappa\left(\sigma_{1}\right) \mathbf{J},
\end{aligned}
$$

where $\kappa\left(\sigma_{1}\right)=\mathbf{R}\left(\sigma_{1}\right) \Lambda_{1} \mathbf{R}^{-1}\left(\sigma_{1}\right)$. This invariance means that if a particular solution $\psi_{0}$ of the NLSE and its corresponding complex functions $r_{0}(\sigma)$ and $s_{0}(\sigma)$ are known, then a new solution can be obtained from Eq. (6). (Recall that the matrix $\mathbf{U}$ is a function of the desired solution $\psi$.) One can then apply the technique on the new solution, as the Darboux transformation also provides the corresponding functions satisfying the linear set (3) through Eq. (5). In this way one can construct iteratively and via only algebraic transformations an entire hierarchy of solutions up to arbitrary order, obtaining the complete range of higherorder MI solutions from the trivial NLSE plane wave solution $\psi_{0}=e^{i \xi}[19]$.

In physical terms, the first-order solution of this type gives the elementary breather of Eq. (2). The $N$ th-order solution constructed from further iteration corresponds to the nonlinear superposition of $N$ such elementary breathers, each associated with unique parameters $\sigma_{k}=$ $\left\{a_{k}, \tau_{0 k}, \xi_{0 k}\right\}$; a distinct modulation frequency (determined by $a_{k}$ ) and temporal and spatial origins $\tau_{0 k}$ and $\xi_{0 k}$ (which depend on initial conditions and can be considered as free parameters).

Although general solutions can be constructed for arbitrary unequally spaced modulation frequencies within the gain band, higher-order MI solutions can also be excited by a single initial modulation frequency, provided that it is below a critical low frequency limit. In particular, for a primary modulation frequency $\Omega, N$ th-order MI $(N \geq 2)$ is excited when $N \Omega<\Omega_{\max }=2$ such that the initial frequency generates harmonics at $\Omega_{k}=k \Omega$, where $k=$ $1, \ldots, N$ and all modes experience gain under the elementary MI gain curve. In terms of the input modulation parameter $a$, this constraint is $N^{2}<(1-2 a)^{-1}$ so that we can readily determine the low frequency limit for higher-order $\mathrm{MI}$ as $\Omega<1$, equivalent to $a>0.375$. The condition between $N$ and $a$ allows the integral order of the solution expected for given input conditions to be readily determined (see Fig. 2 below).

We now apply this analysis to interpret numerical simulations of typical higher-order MI dynamics. Figure 1(a) shows simulation results from integrating numerically the NLSE for initial conditions that do not correspond to the exact mathematical form required for perfect breather growth and decay [10]. We consider an initial field $\psi(\xi=$ $0, \tau)=\left[1+a_{\text {mod }} \cos (\Omega \tau)\right]$, where $a=0.43$ is near the limit of recent experiments studying Peregrine soliton 
evolution, and in the regime $a>0.375$ discussed above where we expect higher-order MI dynamics. In particular, for this $a$, two frequencies $\Omega=0.75\left(=\Omega_{1}\right)$ and $2 \Omega=1.5\left(=\Omega_{2}\right)$ fall within the MI gain band $(\Omega<2)$ so we expect to see $N=2$ higher-order MI evolution.

The figure shows results for two values of modulation amplitude $a_{\text {mod }}$ where we see an initial phase of typical breather evolution (growth along $\xi$ and temporal compression along $\tau$ ), but in both cases evolution beyond the point of maximum amplitude is associated with temporal splitting into subsidiary pulses. Note that the value of $a_{\text {mod }}$ influences both the exact point of temporal compression as well as the nature of subsequent splitting. Although similar dynamics have been previously seen in numerical simulations [6,12], we now use the Darboux transformation to analytically describe this behavior. In particular, we construct through the scheme described above the analytical superposition of the two elementary breathers associated with the frequencies $\Omega_{1}=0.75$ and $\Omega_{2}=1.5$. The constructed solution in this way is shown in Fig. 1(b) for parameters $\sigma_{1}=\{0.430,0,13.41\}, \sigma_{2}=$ $\{0.219,0,18.425\}$ for the low modulation amplitude $a_{\text {mod }}=2 \times 10^{-4}, \quad$ and $\quad \sigma_{1}=\{0.430,0,3.4\}, \quad \sigma_{2}=$ $\{0.219,0,4.47\}$ for the higher modulation amplitude $a_{\text {mod }}=2 \times 10^{-1}$. Here subscripts 1 and 2 refer to the
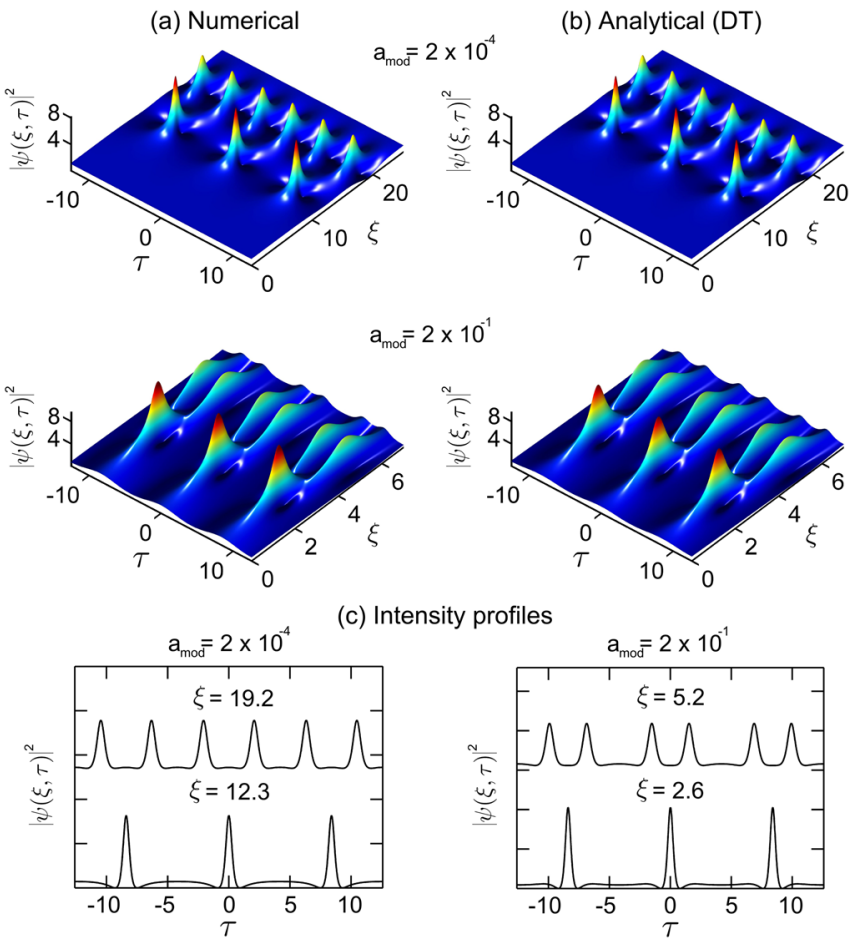

FIG. 1 (color online). Spatiotemporal evolution of a weakly modulated cw field with $a=0.43$ and for values of $a_{\text {mod }}$ as shown. The left column (a) shows results from numerical simulations; the right column (b) shows results from the Darboux transformation (DT) solutions. Results in (c) are line profiles at selected distances for the two values of modulation amplitude. elementary breather solutions associated with frequencies $\Omega_{1}$ and $\Omega_{2}$, respectively.

We first note that the solutions constructed from the Darboux transformation are in excellent agreement with those obtained from the numerical integration of the NLSE. Our analysis also allows us to obtain significantly further physical insight into the dynamics because the construction of the higher-order solutions strongly depends on the relative separation in $\xi$ between the centers of the constituent elementary solutions. When the separation is large, the superposition solution is simply the linear sum of elementary solutions of the form (2). In this case, the first stage of propagation corresponds to that of the primary breather defined by $\Omega_{1}$ followed by a secondary stage that is dominated by the dynamics of the secondary breather defined by $\Omega_{2}$. Such independent dynamics are seen for small modulation amplitudes, and the left-hand panel of Fig. 1(c) shows this clearly by plotting the intensity profiles at the points of maximum amplitude during the initial and secondary evolution phases. Note that because of the nonlinear nature of the superposition of the constituent solutions, the distances $\xi$ of maximum amplitude are close but not identical to the corresponding center parameters of the Darboux transformation given by $\sigma_{1}$ and $\sigma_{2}$.

Significantly, however, provided that the nonlinear coupling is weak such that the splitting can be distinctly observed, the manner in which the higher-order solutions are constructed from elementary solutions suggests that the subsidiary peaks upon splitting can be rescaled to the primary pulse that generates them. Indeed, it is straightforward to show that, for $a>1 / 8$, the breather peaks at the point of maximum amplitude obey an area theorem such that peaks at step $k$ and step $k+1$ are related through $q \psi_{k+1}(t / q)=\psi_{k}(t)$ with the scale parameter $q=$ $\max \left|\psi_{k}\right| / \max \left|\psi_{k+1}\right|>1$. This result is an important link with previous numerical studies of scaling effects in pulse splitting dynamics [14].

On the other hand, for increased input modulation amplitude the nonlinear coupling between the constituent solutions becomes more significant, and it is not possible to consider the evolution as an independent superposition of constituent breathers. This coupling affects the intensity profile of both individual breathers as well as the temporal evolution of the secondary breather phase, and is seen more clearly in the right-hand panel of Fig. 1(c).

For even lower modulation frequencies and MI of increasingly higher orders, simulations show a series of progressive temporal bifurcation dynamics. Yet, even for this case the Darboux transformation can be used to accurately reconstruct and interpret the dynamics. This is illustrated in Fig. 2. We first show in Fig. 2(a) the bifurcation points corresponding to the range of values of $a$ where order $N$ splitting is observed based on the relation between $N$ and $a$ discussed above. This graph is especially useful in allowing the dynamics complexity in terms of $N$ to be 
quantified for a specific input $a$. Figures 2(b) and 2(c) show simulation results for $N=3 \quad(a=0.468)$ and $N=5(a=0.486)$, respectively. For the latter case we also show in Fig. 2(d) the spectral evolution which clearly exhibits a complexity not seen in simple MI. Simulation results in Fig. 2(e) are for $N=6(a=0.4895)$ to show even higher-order splitting. For this case, we also explicitly show the power of the Darboux transformation to reconstruct these dynamics, with the analytic results in Fig. 2(f). All simulations used $a_{\text {mod }}=0.02$.

The above analysis of the complex dynamics in terms of higher-order MI processes has allowed us to design experiments to excite the nonlinear superposition breather solutions using a modulated plane wave optical field injected into optical fiber. Our setup is similar to that of Ref. [8] and consists of a $16 \mathrm{GHz}$ intensity modulated $1550 \mathrm{~nm}$ laser diode of $0.8 \mathrm{~W}$ average power after an erbium-doped fiber amplifier. A phase modulator is also used to inhibit Brillouin scattering. The optical fiber is an $8.34 \mathrm{~km}$ long segment of SMF-28 with group velocity dispersion and third-order dispersion at $1550 \mathrm{~nm}: \beta_{2}=-21.4 \mathrm{ps}^{2} / \mathrm{km}$ and $\beta_{3}=0.12 \mathrm{ps}^{3} / \mathrm{km}$, respectively. The nonlinear parameter $\gamma=1 \mathrm{~W}^{-1} \mathrm{~km}^{-1}$ and the fiber losses are $0.19 \mathrm{~dB} / \mathrm{km}$. The third-order dispersion is included in simulations for completeness but has negligible effect on the dynamics which are well described by the NLSE of
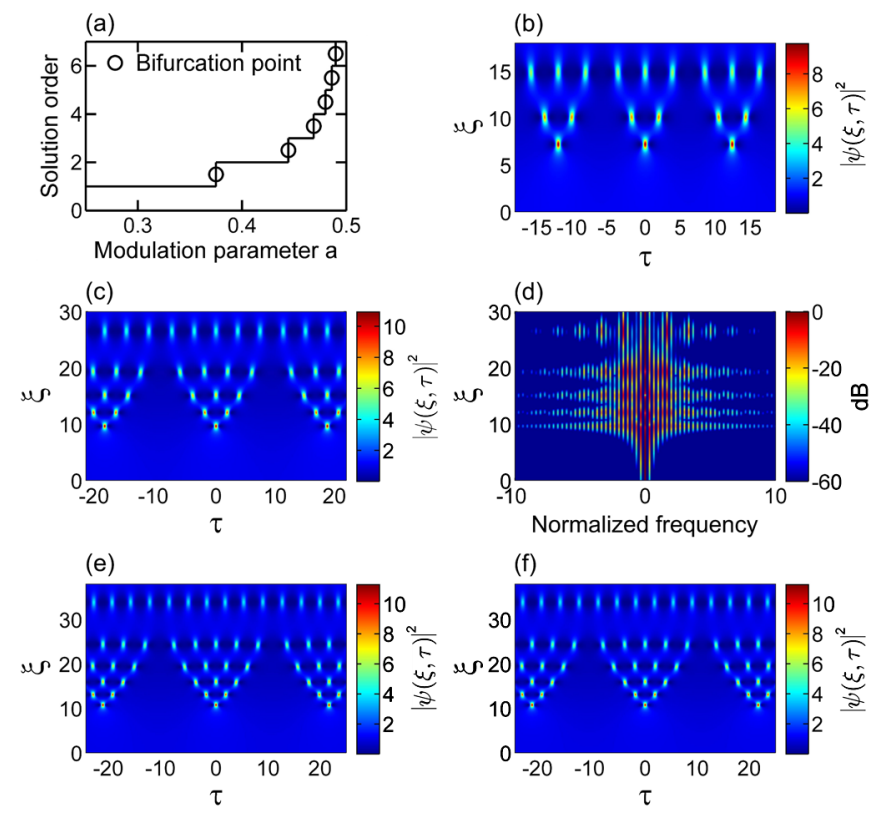

FIG. 2 (color online). Pulse splitting with higher-order MI. (a) Bifurcation points showing the range of $a$ where order- $N$ splitting is observed. (b),(c) NLSE simulations for $N=3$ ( $a=0.468)$ and $N=5(a=0.486)$, respectively. For the latter case (d) shows the associated complex spectral evolution. (e) Simulations for $N=6(a=0.4895)$ to show even higherorder splitting. (f) Corresponding analytical construction using the Darboux transformation for case (e).
Eq. (1). The output signal is characterized in the time domain by means of an ultrafast optical sampling oscilloscope (Picosolve) which, when combined with cutback measurements, allows direct characterization of the longitudinal field evolution. Numerical simulations are based on the dimensional variant of Eq. (1) using experimental parameters. The initial condition is $A(z=0, T)=$ $\sqrt{P_{0}}\left[1+\alpha_{\text {mod }} \cos \left(\omega_{\bmod } T\right)\right]^{0.5}$, where $P_{0}$ is the power of the cw field, $A=\psi \sqrt{P_{0}}, z=\xi /\left(\gamma P_{0}\right), T=\tau T_{0}$, $\omega_{\text {mod }}=\Omega / T_{0}$. and $T_{0}=\left[\left|\beta_{2}\right| /\left(\gamma P_{0}\right)\right]^{1 / 2}$. The parameter $\alpha_{\text {mod }}=0.58$ here is the intensity modulation contrast. Note that with these parameters, the normalized input modulation frequency is $\Omega=0.539$, parameter $a=$ 0.464 , and $N=2 / \Omega=3.7$ consistent with the observed excitation of 3 subpulses at the fiber output.

Our experimental results are shown in Fig. 3(a), and clearly exhibit the expected higher-order MI dynamics with splitting of the initial modulated field into two, then three subpulses with propagation. Numerical simulations including all experimental parameters are shown in Fig. 3(b) and are in excellent agreement with experiment. The analytic construction of the corresponding field using the Darboux transformation is shown in Fig. 3(c). Since the Darboux transformation applies meaningfully only to the ideal NLSE, we do not include loss in the analytic construction, and thus the calculated field displays a higher amplitude than in experiments, but the calculated field nonetheless displays identical temporal splitting to experiment at the fiber output. The analytically constructed third-order solution used $\sigma_{1}=\{0.464,0,4.5\}, \quad \sigma_{2}=\{0.355,0,4.9\}, \quad \sigma_{3}=$ $\{0.173,0,7\}$.

These results represent the first quantitative study of higher-order MI dynamics in any physical NLSE system. The analysis of breather dynamics in terms of the Darboux transformation allows us to naturally interpret the complex evolution at low instability modulation
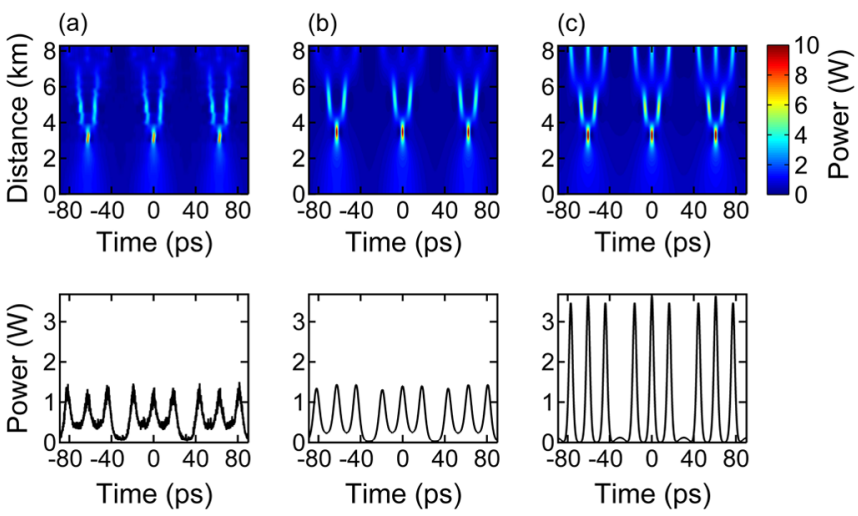

FIG. 3 (color online). Top: Spatiotemporal evolution of $\mathrm{cw}$ modulated field for $a=0.464$. Bottom: Temporal profile at a distance of $8.34 \mathrm{~km}$. (a) Experiments, (b) NLSE simulation, and (c) analytical solution from the Darboux transformation. 
frequencies in terms of the superposition of multiple elementary instabilities governed by distinct dynamical parameter sets. We have shown that higher-order MI dynamics can be readily excited by injecting a modulated plane wave with modulation frequency selected so that its harmonics are also located within the modulation instability gain curve. Our ability to quantitatively describe the measured third-order pulse splitting using the Darboux construction is a remarkable illustration of the power of this analytical method, and we anticipate that these results will motivate its use for similar analysis of wave localization dynamics in other NLSE governed systems such as deep water hydrodynamics, Bose-Einstein condensates, etc. We further expect that signatures of higher-order modulation instability will be found in cases where the entire range of unstable modes spontaneously grows from noise. It should also be clear that the Darboux transformation allows for exactly constructing solutions of very high order which are otherwise extremely difficult to generate through numerical simulations because of prohibitive computational effort. In this context, it is especially important to note that the analytical approach allows us to identify the appropriate initial conditions necessary to generate a particular high-order excitation state; determining the initial conditions would not be possible numerically. This makes the Darboux transformation particularly adapted for the (numerical and experimental) search and decomposition of new forms of instabilities and localization dynamics in various nonlinear regimes including turbulence [16], Anderson localization [20], and selfsimilarity [15]. Finally, we anticipate that the analysis developed may also have important technical applications in providing insights into optimizing the generation of high repetition rate pulse trains and broadband fiber parametric amplifiers.

We thank the Academy of Finland (Research Grants No. 121953, No. 130099, and No. 132279), the graduate school of Tampere University of Technology, the Institut Universitaire de France, the French Agence Nationale de la Recherche projects IMFINI (ANR-09-BLAN-0065) and MANUREVA (ANR-08-SYSC-019), the Conseil
Régional de Bourgogne, and the Australian Research Council (Discovery Project No. DP110102068).

[1] V.E. Zakharov and L. A. Ostrovsky, Physica (Amsterdam) 238D, 540 (2009).

[2] K. B. Dysthe and K. Trulsen, Phys. Scr. T82, 48 (1999).

[3] A. I. Dyachenko and V.E. Zakharov, JETP Lett. 81, 255 (2005).

[4] N. Akhmediev, A. Ankiewicz, and M. Taki, Phys. Lett. A 373, 675 (2009).

[5] N. Akhmediev and V. Korneev, Theor. Math. Phys. 69, 1089 (1986).

[6] J. M. Dudley, G. Genty, F. Dias, B. Kibler, and N. Akhmediev, Opt. Express 17, 21497 (2009).

[7] B. Kibler, J. Fatome, C. Finot, G. Millot, F. Dias, G. Genty, N. Akhmediev, and J. M. Dudley, Nature Phys. 6, 790 (2010).

[8] K. Hammani, B. Kibler, C. Finot, P. Morin, J. Fatome, J. M. Dudley, and G. Millot, Opt. Lett. 36, 112 (2011).

[9] A. Chabchoub, N.P. Hoffmann, and N. Akhmediev, Phys. Rev. Lett. 106, 204502 (2011).

[10] M. Erkintalo, G. Genty, B. Wetzel, and J. M. Dudley, Phys. Lett. A 375, 2029 (2011).

[11] N. Akhmediev and A. Ankiewicz, Solitons, Nonlinear Pulses and Beams (Chapman and Hall, London, 1997).

[12] S. Wabnitz and N. Akhmediev, Opt. Commun. 283, 1152 (2010).

[13] V. B. Matveev and M. A. Salle, Darboux Transformations and Solitons, Series in Nonlinear Dynamics. (Springer Verlag, Berlin, 1991).

[14] S. Sears, M. Soljacic, M. Segev, D. Krylov, and K. Bergman, Phys. Rev. Lett. 84, 1902 (2000).

[15] J. M. Dudley, C. Finot, D. J. Richardson, and G. Millot, Nature Phys. 3, 597 (2007).

[16] F. Dias, A. Pushkarev, and V. Zakharov, Phys. Rep. 398, 1 (2004).

[17] E. A. Kuznetsov, Sov. Phys. Dokl. 22, 507 (1977).

[18] Y. Ma, Stud. Appl. Math. 60, 43 (1979).

[19] N. Akhmediev, V. I. Korneev, and N. V. Mitskevich, Sov. Phys. JETP 67, 89 (1988) [http://www.jetp.ac.ru/cgi-bin/e/ index/e/67/1/p89?a=list].

[20] Y. Krivolapov, S. Fishman, and A. Soffer, New J. Phys. 12, 063035 (2010). 